\title{
Verlernen: Vom alltagsweltlichen zum erziehungswissenschaftlichen Verständnis
}

\author{
Astrid Seltrecht
}

Online publiziert: 10. März 2015

(c) Die Autor(en) 2015. Dieser Artikel ist auf Springerlink.com mit Open Access verfügbar.

Zusammenfassung Verlernen ist als Phänomen im alltagsweltlichen Sprachgebrauch etabliert, aber erziehungswissenschaftlich bislang kaum beachtet worden. Nur wenige Autoren verwenden den Begriff des Verlernens überhaupt, noch seltener ist eine dezidierte und differenzierte Begriffsbestimmung zu finden. Im vorliegenden Beitrag werden zunächst Dilemmata skizziert, die in Erscheinung treten, wenn der alltagsweltliche Begriff des Verlernens als Analysekategorie in empirischen Arbeiten verwendet wird. Die kritische Re-Analyse einer dieser Arbeiten aber zeigt zugleich ein Arbeitsprogramm für die Ergründung von Phänomenen des Verlernens auf. Einer dieser Aufgaben - die Entwicklung einer Heuristik des Verlernens aufgrund einer Literaturanalyse - nimmt sich der vorliegende Beitrag in einem ersten Schritt an.

\section{Schlüsselwörter Erfahrung $\cdot$ Lernen $\cdot$ Verlernen $\cdot$ Nichtlernen}

\begin{abstract}
Unlearning has been established as a phenomenon in common parlance, but has been neglected by educational science so far. Very few authors use the term "unlearning", even rarer is a clear, precise and sophisticated definition. This article starts with the dilemmata appearing when using the term as a category of analysis in empirical studies. The critical re-analysis of one of these studies yet evokes a work programme designed to explore the phenomena of unlearning. As a first step, this article deals with one of these tasks: developing heuristics of unlearning on the basis of a literature review.
\end{abstract}

Keywords Experience $\cdot$ Learning $\cdot$ Unlearning $\cdot$ Non-learning

\footnotetext{
Jun. Prof. Dr. A. Seltrecht $(\square)$

Institut für Berufs- und Betriebspädagogik, Otto-von-Guericke-Universität Magdeburg,

Zschokkestraße 32, 39104 Magdeburg, Deutschland

E-Mail: astrid.seltrecht@ovgu.de
} 


\section{Einleitung: Alttagsweltliche Verwendung des Begriffs „Verlernen“ in der Erziehungswissenschaft}

Lernen wird in vielfältiger Weise diskutiert, z. B. hinsichtlich der Perspektive und der professionellen Bezugnahme (bildungspolitisch, erziehungswissenschaftlich, pädagogisch oder institutionell/organisatorisch) oder hinsichtlich der verschiedenen Lernkontexte, in denen - gleichsam im Verbund über die gesamte Lebensspanne hinweg - das lebenslange Lernen stattfindet (z. B. formal, non-formal, informell), oder hinsichtlich der verschiedenen Lehrformen, die das Lernen einer Zielgruppe ermöglichen sollen (z. B. forschendes Lernen, problemorientiertes Lernen, situiertes Lernen, fallbasiertes Lernen). Die Ausrichtung von Lernprozessen, seien sie nun pädagogisch intendiert oder nicht pädagogisch intendiert, ist i. d. R auf eine Zunahme (z. B. von Wissen oder Können) oder eine Entwicklung (z. B. der Identität oder von Kompetenzen) gerichtet. Neben dieser eher positiven Thematisierung eines (Neu-)Lernens, die wir in der Bildungspolitik, aber auch in der Erziehungswissenschaft und der Pädagogik vorfinden, existiert im alltagsweltlichen Verständnis auch das Wissen um Formen des Verlernens, Umlernens und Nichtlernens. Gerade Verlernen oder Vergessen ist uns allen durch (berufs-)schulische und/oder universitäre Erfahrungen bekannt. Wer kann heute beispielsweise noch erklären, was unter der Strukturisomerie bei Alkanen zu verstehen ist (nämlich dass es Moleküle gibt, die die gleiche Summenformel, aber unterschiedliche Strukturformeln haben - ein Thema aus dem Chemieunterricht der 10. Klasse), oder - aus dem Biologieunterricht derselben Jahrgangsstufe - die Bausteine der menschlichen DNA benennen (Phosphorsäure, Desoxyribose, Adenin, Thymin, Cytosin, Guanin)? Diese ,dunkle“ Seite von Lehr-Lern-Prozessen wird zumindest bei der Thematisierung des lebenslangen Lernens ausgeblendet. Und wenn der Begriff des Verlernens in erziehungswissenschaftlichen Arbeiten dennoch auftaucht, dann meist ohne dezidierte und differenzierte Bestimmung (Kade und Seitter 2007b, S. 316 f.; Maier-Gutheil 2009, S. 17, 34, 115; Dinkelaker 2011, S. 133; Richter und Friebertshäuser 2013, S. 323). Stattdessen scheint diese Begriffsverwendung auf eine geteilte alltagsweltliche Vorstellung von Verlernen zu rekurrieren. Dieses Verständnis deckt sich mit der im Duden skizzierten Wortbedeutung: „(etwas Erlerntes, Gewusstes, Gekonntes) allmählich immer weniger, schließlich gar nicht mehr beherrschen“" (www.duden.de). Deutlich wird bei dieser Auslegung, was allmählich verlernt wird, nämlich etwas zuvor „Gewusstes“, im Sinne von Wissen, bzw. „Gekonntes“, im Sinne von Fertigkeiten, das bzw. die man sich in vorangegangenen Lernprozessen angeeignet hat. Offen bleibt jedoch, wie sich dieses Verlorengehen vollzieht. Wir haben es hier alltagsweltlich mit der Unterscheidung von Form (,Wie wird verlernt?") und Inhalt („Was wird verlernt?") bzw. mit Verlernen als Lernprozess vs. Verlernen als Lernergebnis zu tun.

Ebenfalls an das alltagsweltliche Verständnis vom Verlernen anknüpfend, wurde in einem biografieanalytisch angelegten Forschungsprojekt (Nittel und Seltrecht 2013) Verlernen als eines von insgesamt vier Lernmodi (Neulernen, Umlernen, Verlernen, Nichtlernen) konzipiert. Hier wurde versucht, die der Alltagswelt entlehnten Begriffe einerseits für die grundlagentheoretische Erforschung eines lebenslangen Lernens und andererseits methodisch für die Analyse empirischen Datenmaterials zu nutzen, ohne jedoch eine theorieorientierte und eine empirisch fundierte Re-Inter- 
pretation des Begriffs „Verlernen“ vorzunehmen (z. B. Nittel 2010, S. 95, 99, 2011, S. 84 f., 2013, S. 111, 145). Das Anlegen dieser Begriffe als Analysekategorien - wie im Projektkontext unternommen und für weitere Analysen vorgeschlagen (z. B. Siewert 2014) - offenbart allerdings Schwächen.

\section{Dilemmata der Verwendung des alltagsweltlichen Begriffs ,Verlernen“ in der erziehungswissenschaftlichen Forschung}

Diese noch zu bearbeitenden Herausforderungen sollen anhand eines Fallbeispiels - einer Familiengeschichte, die aufgrund der Brustkrebserkrankung der Mutter (Uta Berger) erhoben wurde - verdeutlicht werden: Im Rahmen der Erhebung der Familiengeschichte durch die Autorin tragen die Eheleute Georg und Uta Berger und ihre gemeinsame 18-jährige Tochter Lisa Ereignisse jeweils abwechselnd aus ihrer subjektiven Perspektive zusammen. Im Verlauf des Familiengesprächs wird deutlich, dass Lisa zu der Zeit, in der ihre Mutter durch eine Chemotherapie ihre Haare verlor (Lisa war damals 13 Jahre alt), nicht mehr nachts ins elterliche Schlafzimmer kommen wollte:

Lisa wiederholt, dass sie darüber nichts sagen könne, räumt aber ein, dass sie nur wisse, dass sie dann nicht mehr zur Mutter ins Bett habe kommen wollen („Das weiß ich nicht. Ich weiß nur, dass ich des einfach dann nicht machen wollte"). Nach Utas Rezeptionssignal verhindert Lisa, dass es zu einer Interaktionskrise kommt, indem sie eigentheoretische Überlegungen anstellt und vermutet, dass die Suche nach einem neuen Kontrollhandlungsschema zu einer langfristigen Verhaltensänderung geführt haben könnte. (...) Lisa gibt in ihren Ausführungen zu verstehen, dass sie sich mit dem neuen Kontrollhandlungsschema des Sich-allein-Beruhigens im eigenen Zimmer gleichzeitig das Aufsuchen der Eltern in der Nacht ,abgewöhnt“ habe. Von „Abgewöhnen“ spricht man in der Regel eher bei „schlechten“ Gewohnheiten. Das würde bedeuten, dass Lisa erst etwas gelernt und es dann korrigiert, das Fehlerhafte also gleichsam verlernt hätte. Wird also der subjektiven Bewertung von Lisa und der alltagsweltlichen Wortbedeutung gefolgt, handelt es sich um ein Verlernen. (...) Geht man aus Beobachterperspektive davon aus, dass es für kleinere Kinder jedoch mehr als legitim ist, nachts bei den Eltern Schutz und Geborgenheit zu suchen, lässt sich das Lernen auch als - altersentsprechend notwendiges - Neulernen fassen, denn erstmals in ihrem Leben weiß Lisa sich nun selbst zu beruhigen. Unter entwicklungspsychologisch-normativer Perspektive ist sie mit 13 Jahren jedoch eigentlich schon zu alt, um nachts noch immer die Nähe der Eltern zu benötigen; bis zu diesem Zeitpunkt hatte sie ein altersentsprechendes Verhalten also noch nicht gelernt. Wird jedoch auf die Veränderung selbst - von einem Kontrollhandlungsschema zu einem anderen Kontrollhandlungsschema - fokussiert, muss von einem Umlernen gesprochen werden. Es ist also zunächst jeweils die Perspektive zu klären, aus der heraus der Lernmodus beschrieben werden soll (Seltrecht 2013, S. 174 ff.). 


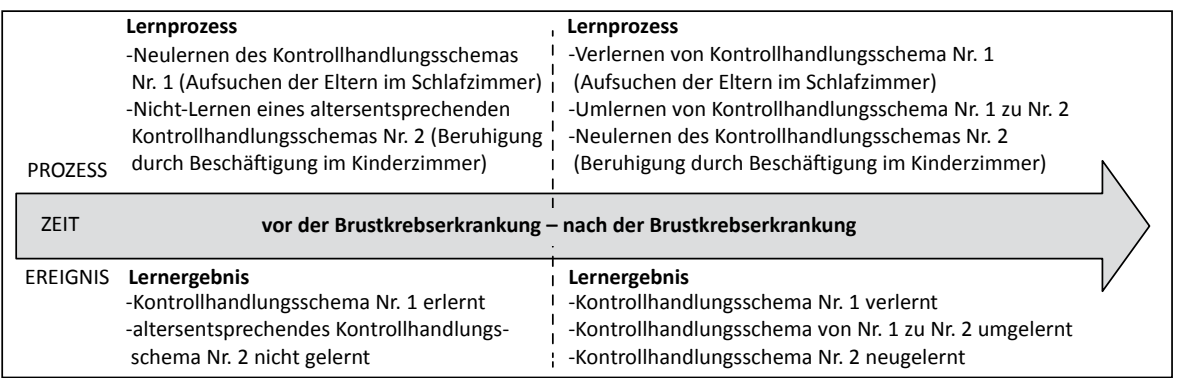

Abb. 1 Lisa Bergers Lernprozesse und Lernergebnisse vor und nach der zweiten Brustkrebserkrankung ihrer Mutter Uta (eigene Darstellung)

Die analytisch-methodische Frage, vor der der Forscher bzw. die Forscherin an dieser Stelle steht, ist die, zu entscheiden, um welchen Lernmodus es sich bei Lisa Berger zum Zeitpunkt der Brustkrebserkrankung der Mutter handelt: Handelt es sich zum Zeitpunkt der Diagnose um ein neu gelerntes erstes Kontrollhandlungsschema oder um ein (noch) nicht gelerntes altersgerechtes (zweites) Kontrollhandlungsschema? Handelt es sich im Verlauf der Brustkrebserkrankung um ein Verlernen des ersten, zuvor entwickelten Kontrollhandlungsschemas, um ein Umlernen von einem ersten zu einem zweiten Kontrollhandlungsschema oder um ein Neulernen eines neuen Kontrollhandlungsschemas? Das derzeitige Dilemma der bislang von außen an die Fälle angelegten alltagsweltlichen Begriffe „Neulernen“, „Verlernen“, „Umlernen“ und „Nichtlernen“ illustriert exemplarisch Abb. 1 anhand der skizzierten Lernphänomene bei Lisa Berger.

Die Festlegung, um welche Art des Lernens es sich jeweils handelt, scheint sich aus verschiedenen Koordinaten zu bestimmen: aus dem Lernprozess und dem Lernergebnis sowie aus der Bewertung von Lernprozess und Lernergebnis zu einem konkreten Bewertungszeitpunkt vor dem Hintergrund ehemaliger eigener oder fremder Erwartungen an Lernprozess und Lernergebnis als Bewertungsmatrix.

Die Herausforderungen in der Analyse biografischen Materials ergeben sich schließlich auch dadurch, dass nicht nur der Forscher bzw. die Forscherin aus Beobachterperspektive auf die Erfahrungsaufschichtung schaut, also jeweils entscheiden muss, zu welchem Zeitpunkt ein Lernprozess und ein Lernergebnis von ihm/ ihr bewertet werden, sondern dass auch der Erzähler oder die Erzählerin zwischen erzählter Zeit und Erzählzeit wechselt, so dass zu subjektiv gesetzten Zeitpunkten innerhalb der konstruierten Biografie Lernprozesse dargestellt und Lernergebnisse vor dem Hintergrund eigener und/oder fremder Erwartungen bewertet werden.

\section{Vom alltagsweltlichen zum erziehungswissenschaftlichen Verständnis eines Verlernens - Skizzierung eines Arbeitsprogramms}

Die Versuche, den Begriff des Verlernens für ein Verstehen lebenslanger Lernprozesse empirisch zu ergründen und gleichzeitig als Analysekategorie bei der Auswertung empirischen Materials zu verwenden, können - als Themendimensionierung 
verstanden - letztlich dennoch positiv genutzt werden. So zeigen die ersten Analyseerfahrungen, dass nun weitere Aufgaben anstehen:

1. Es muss eine komparative Auseinandersetzung mit den Begriffen „Verlernen“ und ,Vergessen “ erfolgen, vor allem, weil der letztgenannte Begriff sowohl alltagsweltlich als auch fachwissenschaftlich in psychologischen und sozialwissenschaftlichen Theorien verwendet wird.

2. Es ist eine äußere Abgrenzung des Begriffs „Verlernen“ von anderen Lernmodi vorzunehmen, um den Begriff trennscharf zu machen.

3. Weiterhin ist eine innere Differenzierung des Begriffs notwendig, um die ihm innewohnenden Dimensionierungen offenzulegen.

4. Und auf der Grundlage einer theoretischen Auseinandersetzung mit bereits vorliegenden Arbeiten ist zunächst einmal eine Heuristik zu entwickeln, die die noch ausstehende empirische Analyse von Phänomenen des Verlernens (im Sinne einer äußeren Abgrenzung und inneren Differenzierung) für einen empirisch fundierten erziehungswissenschaftlichen Begriff unterstützt.

Eine erste Spurensuche hat Arbeiten zutage gefördert, die einmal ein Vergessen, ein andermal ein Verlernen thematisieren und Verlernen mal auf der Ebene des Individuums, mal auf der Ebene einer Organisation oder gar auf der kollektiven Ebene einer Gesellschaft ansiedeln. In einigen Texten werden Phänomene des Verlernens positiv, da erwünscht, in anderen hingegen negativ, da unerwünscht, bewertet. Alle diese Arbeiten stehen völlig losgelöst nebeneinander - Bezüge zu anderen Arbeiten, die ein Verlernen oder Vergessen thematisieren, werden nur in Ausnahmefällen hergestellt. Der vorliegende Beitrag versucht, diese in der erziehungswissenschaftlichen, sozialwissenschaftlichen und bildungsphilosophischen Literatur sowie in subjektiven Erfahrungsberichten gefundenen Thematisierungen miteinander ins Verhältnis zu setzen und auf dieser Grundlage eine erste Heuristik des Verlernens zu entwickeln. Hierfür wurden die in den einzelnen Beiträgen anklingenden Verständnisse eines Verlernens so weit verallgemeinert, dass die einzelnen entwickelten Kategorien schließlich innerhalb einer Heuristik abgebildet werden können.

\section{Lernen und Verlernen unter dem Fokus der Erfahrungsaufschichtung}

Der Entwurf einer Heuristik des Verlernens beruht - und das ist, da nicht allein auf erziehungswissenschaftliche Arbeiten zurückgegriffen wird, notwendig zu betonen auf der Grundlage eines genuin pädagogischen Lernverständnisses, nach dem Lernen nicht nur auf Erfahrung fußt, sondern Erfahrung ist (Meyer-Drawe 2008, S. 15, 214). Hierbei ist zu beachten, dass wir einerseits Erfahrungen machen, diese uns andererseits aber auch widerfahren können. Pädagogische Theorien thematisieren - entgegen dem gesellschaftlichen Mainstream, der Lernen als Anpassung an z. B. gesellschaftliche, institutionelle, technische Entwicklungen versteht - immer auch Irritationen, Störungen oder Verzögerungen des Lernens. Und sie beinhalten - im Gegensatz zu psychologischen Lerntheorien - immer auch eine philosophische Perspektive. Es muss jedoch konstatiert werden, dass gerade im 20. Jahrhundert in der Pädagogik 
eher psychologische als genuin pädagogische Lerntheorien dominierten (Petzelt 1961; Koch 1988, 1991; Göhlich und Zirfas 2007; Göhlich et al. 2007; Buck 1989).

Lernen als Erfahrungsaufschichtung ist, entgegen den Prophezeiungen der Hirnforschung, „nicht durchschaut“: Wir können lediglich Lernergebnisse feststellen, im Vollzug des Lernens als Prozess ist es uns bei uns selbst und in der Bestimmung von Lernprozessen anderer entzogen. Es handelt sich gleichsam um ein „menschliches Rätsel“" (Meyer-Drawe 2008, S. 32):

Lernen (ist) nach wie vor nur sehr notdürftig verstanden (...). Dies hängt mit dem grundsätzlichen Problem zusammen, dass ich beim Lernen auf das Was und nicht auf das Wie gerichtet bin. Zwar gibt es zahlreiche, auch bewährte Definitionen, aber was genau beim Lernen geschieht, ist nach wie vor ungeklärt (ebd., S. 33).

Und aus pädagogischer Perspektive ist Lernen an die Konstruktion eines Sinns gebunden. Letztlich wird auch dadurch die Ergründung von Lernprozessen erschwert, denn „Gründe dafür, dass jemand etwas als etwas lernt“", lassen sich nicht neurobiologisch abbilden - „Bedeutungen sind keine empirischen Größen“ (ebd., S. 31).

Wie sind, bei dieser recht schwierigen Ausgangslage, nun Lernen und Verlernen unter der Perspektive der Erfahrungsaufschichtung aufeinander bezogen? Das wohl erste und bekannteste Beispiel in der Literatur, das ein Lernen und Verlernen anspricht, ist das Höhlengleichnis von Platon:

Stelle dir die Menschen vor in einem unterirdischen, höhlenartigen Raum, der gegen das Licht zu einen weiten Ausgang hat über die ganze Höhlenbreite; in dieser Höhle leben sie von Kindheit, gefesselt an Schenkeln und Nacken, so daß sie dort bleiben müssen und nur gegen vorwärts schauen, den Kopf aber wegen der Fesseln nicht herumdrehen können; aus weiter Ferne leuchtet von oben her hinter ihrem Rücken das Licht des Feuers (...). Sie gleichen uns! Denn sie sehen zunächst von sich und den anderen nichts außer den Schatten, die von dem Feuer auf die gegenüberliegende Mauer geworfen werden (Platon Politeia 514a).

Platon, der das Höhlengleichnis im Gespräch mit Glaukon entwickelt, fragt dann, was passieren würde, wenn einer der Gefesselten von seinen Ketten befreit werden würde und zunächst den Kopf wenden, später gar ins Licht treten könne. „Würde er da nicht in Verlegenheit sein und glauben, was er vorher erblickt, sei wirklicher als das, was man ihm jetzt zeige?" (Platon Politeia 514c). Der nun langsam neu zu konstruierende Welt- und Selbstbezug erfolgt prozesshaft. Das Verlernen vollzieht sich also durch Erfahrungsaufschichtung, die das vormals Gelernte überlagert bzw. revidiert. Dieser Prozess verbindet demnach verschiedene Lernmodi: ein Neulernen, das schmerzlich ist, und ein Verlernen, d. h. das Aufgeben bisheriger Konstruktionen der Welt und des Selbst. Aber:

Dieser Aufbruch zum Licht hat zunächst gar nichts von einem Jubel über die Begegnung mit einer Idee als solcher. Der Betroffene wird im Gegenteil in mehrfacher Hinsicht zum Opfer seiner Befreiung. Ins Sonnenlicht zu blicken blendet ihn und schmerzt seine Augen. Der Blick zurück in die Höhle ist vom Nachflimmern beeinträchtigt, so dass er weder die Idee des Guten schauen noch 
sich in den Schutz der bloßen Meinung und sinnlichen Wahrnehmung zurückziehen kann. Dieser Zustand der Schwebe markiert den Anfang des Lernens: Das Neue wird noch nicht verstanden, dem Alten wird nicht mehr vertraut (Meyer-Drawe 2008, S. 152).

Der Prozess vom (Neu-)Lernen des Neuen und Verlernen des Alten lässt sich gleichsam als Umlernen bezeichnen. Hier zeichnen sich dann auch erste Bedingungen für ein Verlernen ab: Nur wenn zuvor bereits etwas gelernt wurde, ist ein Verlernen möglich. Und ein Verlernen wird verhindert, wenn ein Neulernen ausbleibt, gleichsam nicht gelernt wird. Oder mit Bezug auf das Höhlengleichnis: Wenn niemand die Fesseln löst, so dass der Blick gewendet werden kann und neue Erfahrungen gemacht werden können, wird ein Verlernen - durch das Ausbleiben des Prozesses des Neulernens - verunmöglicht. Die Aufschichtung von Erfahrungen (Dewey 1994, 1995; Buck 1989; für die Bedeutung der Erfahrungsaufschichtung für die qualitative Forschung vgl. auch Schütze 1981) spiegelt sich also direkt in den verschiedenen Lernmodi wider.

Der Bezug von Lernen und Verlernen wird aber nicht nur von der Erfahrungsaufschichtung an sich beeinflusst, sondern auch von der Art und Weise, wie der Austausch des Neuen mit dem Alten erfolgt bzw. wie zugänglich und wie beeinflussbar durch uns er jeweils ist. Mit der Unterscheidung von Lernebenen (Bateson 1985) lassen sich unterschiedliche Bezüge zwischen Lernen und Verlernen ausmachen: Auf der Ebene 0 wird gelernt, ohne zu verlernen. Das Aufnehmen von Lerngegenständen erfolgt sozusagen vor dem Hintergrund einer tabula rasa. Eine Reibung zwischen Altem und Neuem ist ausgeschlossen. Bei einem Lernen auf Ebene 0 handelt es sich also um eine ,einfache Informationsaufnahme von einem äußeren Ereignis, dergestalt, daß ein ähnliches Ereignis zu einem späteren (und geeigneten) Zeitpunkt dieselbe Information übermitteln wird: Ich ,lerne“ von der Werkssirene, daß es zwölf Uhr ist" (Bateson 1985, S. 368). Das Lernen auf dieser Ebene ist zudem Grundlage für Lernprozesse auf allen weiteren Ebenen.

Auf der Ebene I - der das Lernen auf Ebene 0 vorangegangen ist - wird in dem Maße gelernt, wie das Alte durch ein Neues ersetzt wird. Es handelt sich also um eine „Veränderung im ,Lernen null““ (ebd., S. 371). Diese Veränderung beinhaltet, dass wir zwischen dem Alten und dem Neuen unterscheiden können (ebd., S. 373). Wir können bei diesem Konglomerat aus Neulernen und Verlernen bzw. - beides im Prozess betrachtend - Umlernen nicht hinter die einmal gemachte Erfahrung des Lernens auf Ebene 0 zurückfallen. Die Komplexität des Lernens auf Ebene I im Vergleich zur Ebene 0 veranschaulicht Bateson, Heraklit paraphrasierend, mit einem einfachen, aber eindrücklichen Beispiel, wenn er schreibt: „Kein Mann kann zweimal mit demselben Mädchen zum ersten Mal ins Bett gehen“ (ebd., S. 373). Wir können also lediglich zwischen einem ersten Mal (welcher Erfahrung auch immer) und den weiteren Malen unterscheiden, also zunächst gemachte Erfahrungen durch neue Erfahrungen im Prozess des Neulernens und Verlernens modifizieren.

Auf der Ebene II wird hingegen über das Lernen und Verlernen etwas gelernt bzw. verlernt, 
das heißt zum Beispiel in der Lage zu sein, zu verlernen, nur dann zu verlernen, wenn Verunsicherung der alten Routinen und hilfreiches Angebot neuer Routinen Hand in Hand gehen. Denn solange man nur verlernt, wenn man verunsichert wird, agiert man im Kontext von „Macht" und damit in einem Kontext, der kaum Möglichkeiten einräumt, jene Unterscheidung zwischen Information und Wissen zu treffen, die für eine Selbstbeobachtung des Lernens und damit ein Lernen des Lernens wesentlich ist. Die Ebene II impliziert daher zunächst einmal ein Verlernen auch des Verlernens und damit eine Verstärkung der Möglichkeit, bei den bisher bewährten Strategien und Taktiken der Abweichungsvermeidung zu bleiben (Baecker 2003, S. 193).

Auf dieser Ebene werden also die Konsequenzen eines Lernens und Verlernens kritisch reflektiert. Gewiss ist nun auch, dass eine Umkehr eines einmal vollzogenen Lern- bzw. Verlernprozesses nicht einfach realisierbar ist. Immer sind mit den Prozessen des Neulernens auch Verlernprozesse verbunden, deren Reichweite vorher kaum abschätzbar ist - nur dass ein Lernen Auswirkungen auf ein Verlernen nimmt, ist gewiss.

Auf der Ebene III ist es dann möglich, den auf Ebene II erkannten Prozess des Lernens und Verlernens nicht nur kritisch zu beobachten, sondern auch zu modifizieren. Es wird also „nicht nur gelernt und verlernt, wie man lernt und verlernt, sondern es werden Veränderungen im Lernen und Verlernen vorgenommen“ (Baecker 2003, S. 194) (Abb. 2). Aus einer Menge von Alternativen des Lernens und Verlernens kann ausgewählt werden, um Neues zu lernen und Altes $\mathrm{zu}$ verlernen.

\section{Eine erste Differenzierung des Verlernens bezüglich Bewusstheit und pädagogischer Absicht}

Werden nun einzelne Beschreibungen und Belege für ein Verlernen aus der Literatur herangezogen, so zeigt sich, dass Verlernen zum einen als urwüchsiger Vorgang gesehen wird, der en passant abläuft, und zum anderen als bewusst zu beeinflussender Prozess verstanden wird, indem konstatiert wird, dass Verlernen

Abb. 2 Das Verhältnis von Lernen und Verlernen vor dem Hintergrund der Lernebenen nach Gregory Bateson (1985) und der Ausführungen von Dirk Baecker (2003) (eigene Darstellung)

\begin{tabular}{|l|}
\hline $\begin{array}{l}\text { Ebene III } \\
\text { Modifizierung des Lernens und Verlernens, wie sich das Verlernen des Alten durch das } \\
\text { Neulernen des Neuen vollzieht }\end{array}$ \\
$\begin{array}{l}\text { Ebene II } \\
\text { Lernen und Verlernen, wie sich das Verlernen des Alten durch das Neulernen des Neuen } \\
\text { vollzieht }\end{array}$ \\
$\begin{array}{l}\text { Ebene I } \\
\text { Verlernen des Alten durch das Neulernen des Neuen }\end{array}$ \\
$\begin{array}{l}\text { Ebene } 0 \\
\text { Neulernen erfolgt; Verlernen nicht möglich, da noch nichts zum Verlernen vorhanden ist }\end{array}$ \\
\hline
\end{tabular}


aktiv gefördert werden müsse oder - im umgekehrten Fall - ihm aktiv entgegenzugetreten sei. Diese Variationen werden im Folgenden kurz vorgestellt. Sie erheben keinesfalls den Anspruch auf Vollständigkeit, verdeutlichen aber die Notwendigkeit, sich zukünftig in der Erziehungswissenschaft auch diesen Phänomenen zuzuwenden.

\subsection{Verlernen en passant}

Ebenso wie wir ein Lernen en passant kennen, gibt es auch die Möglichkeit eines Verlernens en passant - fast unbemerkt, ohne unser Zutun und ohne jegliche pädagogische Absicht. $\mathrm{Zu}$ fragen ist allerdings, wo und wann es beginnt: Inwieweit bzw. wie sicher in der Abrufbarkeit und Anwendung muss ein Wissen und Können zuvor gelernt worden sein, damit es dann beiläufig verlernt werden kann? Klaus Prange schreibt beispielsweise: „Schon beim Anhören eines Vortrags wie beim Lesen einer Buchseite verschwindet und wird unsicher, was wir eben gehört oder gelesen haben. Wir behalten ja einen Text nicht Wort für Wort im Gedächtnis“" (2006, S. 233). Prange verwendet in seinen Ausführungen den Begriff des Vergessens, nicht den des Verlernens. Hier zeigt sich die eingangs im Arbeitsprogramm zur Erforschung des Verlernens skizzierte Notwendigkeit der komparativen Auseinandersetzung mit den Begriffen „Verlernen“ und „Vergessen“. Auch Bateson (1985) beschreibt ein Phänomen des Verlernens bzw. des Vergessens, ohne jedoch diese Begriffe zu verwenden: „Und es gibt das Erlöschen oder den Verlust von Gewöhnung, die als Ergebnis einer mehr oder weniger großen Lücke oder einer anderen Unterbrechung in der Abfolge von Wiederholungen des Reizereignisses auftreten können“ (S. 372). Darüber hinaus zeigt Prange auf, dass sich Vergessen nicht nur auf der individuellen Ebene, sondern auch auf kollektiver Ebene vollzieht:

Wir können nicht leben, ohne zu vergessen, als Einzelne nicht, und auch nicht als Gruppe und Familie, als Volk und Nation. Das Vergessen gehört gewissermaßen zu unserer Natur. Wir brauchen uns nicht einmal besonders darum zu bemühen. Im Fortgang dessen, was geschieht und was wir tun, verlieren sich die Ereignisse und Erlebnisse. Sie verschwimmen gleichsam, und so bedarf es einer besonderen Anstrengung, um uns zu erinnern und wieder vor Augen zu führen, wie es gewesen ist. Das Vergessen ist, wie Martin Heidegger in „Sein und Zeit" ausgeführt hat, elementarer als das Erinnern. Denn das Erinnern ist nur möglich „auf dem Grunde des Vergessens“ (Prange 2006, S. 233).

Das Erinnern, das Prange hier anspricht, ist dem Vergessen also immer nachgeordnet. Aber, und darin liegt die Bedeutung des Erinnerns, nur das Erinnern zeigt uns unser Vergessen auf: „Der Vollzug selbst des Vergessens ist vom Vergessenen nicht zu erfassen, diskontinuierlich und unterhalb der Schwelle des Bewußtseins entfaltet es seine produktiv-zerstörerische Wirkung" (Giese 1998, zit. nach Meyer-Drawe 2008, S. 175). Dieses Erinnern wird zudem meist von außen, von anderen veranlasst. Erst sie machen uns auf unser Vergessen aufmerksam (vgl. Meyer-Drawe 2008, S. 175).

\subsection{Intentionales Verlernen}

Lässt sich Verlernen auch bewusst bewirken oder verhindern? Zwei Ausprägungen dieser Kategorie werden im Folgenden skizziert. 


\subsubsection{Verlernen bewirken wollen}

Mit Bezug auf die Atomwaffenabwürfe über Hiroshima und Nagasaki schreibt Meyer-Drawe, dass ein kollektives Verlernen unmöglich sei: „Die bedrohliche Dynamik der technischen Entwicklungen besteht darin, dass Menschen nicht willentlich verlernen können. Erfindungen können nicht einfach ungeschehen gemacht werden“ (2008, S. 59). Ihre Ausführungen beziehen sich hier auf den Tatbestand, dass wir hinter die einmal gemachten Erfahrungen nicht zurückfallen können. Nach ihrer Einschätzung ist ein aktives Verlernen-Wollen nicht möglich. Dennoch finden wir in der Literatur Beispiele, in denen ein Verlernen bewusst angestrebt wird. In der Kunst etwa gibt es über die Zeit hinweg immer wieder Beispiele dafür, dass jemand verlernen möchte bzw. sollte. So versuchte der Bildhauer Pierre Giraud in den 1980er Jahren, seine ,akademische Routine zu verlernen“ (Ritter 2013, o. S.). Und auch der spätere Lebensentwurf von Hannah Arendt enthält fast schon ein biografisches Programm des Verlernens (Knott 2011).

\subsubsection{Verlernen verhindern wollen}

Klaus Prange, der das Phänomen des Vergessens bereits beim Hören eines Vortrags oder Lesen eines Buches in Anschlag bringt, da nicht jeder Satz verinnerlicht werde, vielmehr etliche von ihnen sogleich vergessen würden, zeigt auf, wie ein Vergessen verhindert werden kann: Wir ,schaffen uns Merkpunkte, machen uns Notizen oder unterstreichen, was uns wichtig erscheint. Warum? Um nicht zu vergessen und für die Zukunft festzuhalten, was wir vielleicht einmal gebrauchen und beachten wollen“ (Prange 2006, S. 233). Übung, Training, Wiederholung, Anwendung sind die Strategien, die ein Verlernen verhindern helfen. Zeitgeschichtlich ist das Verhindern eines Verlernens nichts Neues: So habe der Philosoph Antisthenes (444 bis ca. 368 v. Chr.) auf die Frage, was jemand am nötigsten hätte zu lernen, geantwortet: „,Dem Verlernen vorzubeugen“" (Laertius 1990, S. 298). Neben diesen Bestrebungen, ein Verlernen auf der Ebene des Individuums zu verhindern, finden sich in der Literatur auch Beispiele dafür, dass Organisationen Verlernen verhindern wollen. So schreibt der Kulturtheoretiker und -analytiker Dirk Baecker (2003): „Die kompetente Organisation weigert sich, zu verlernen, was sie bereits kann. Und deswegen, so unsere Ausgangshypothese, ist sie so erfolgreich darin, nicht zu lernen. Sie weiß etwas, was diejenigen, die sie zum Lernen bringen wollen, nicht wissen“" (S. 179). Diese Verhinderung eines Verlernens von bisher Gelerntem erklärt sich mit den Aufgaben von Organisationen, die nur im geschützten Rahmen, ohne Reize aus der sich ständig wandelnden äußeren Umwelt bearbeitet werden können (ebd., S. 181 f.).

\subsection{Pädagogisch intendiertes Verlernen}

Pädagogisches Handeln ist immer von der Absicht gekennzeichnet, Lernen aufseiten der Zielgruppe zu ermöglichen. Die pädagogische Differenz und die Unverfügbarkeit des Subjekts für andere führen dazu, dass erst in der Rückschau konstatiert werden kann, ob und in welcher Form die pädagogischen Ziele umgesetzt werden konnten obgleich auch dann nicht sicher ist, ob die Ergebnisse auf das pädagogische Handeln 
oder auf andere Prozesse und Einflüsse zurückzuführen sind. Zwei Variationen eines pädagogisch intendierten Verlernens lassen sich voneinander abgrenzen.

\subsubsection{Verlernen pädagogisch bewirken wollen}

Das pädagogisch beabsichtigte Verlernen finden wir oft in denjenigen pädagogischen Handlungsfeldern, wo Abweichungen von einer „Norm“ (Leistungen, Gesundheitszustand, soziale Integration) pädagogisch bearbeitet werden: z. B. Rauchentwöhnung, Ernährungsberatung, Gewaltpräventionstrainings. Diese Form wird, so scheint es, immer dann relevant, wenn das zuvor Gelernte nicht der pädagogischen Norm entspricht. Historisch überliefert ist etwa das Beispiel des Malers und Lehrers Jacques-Louis David (1748-1825), der von seinen Schülern forderte, das vormals Gelernte zu verlernen (Ritter 2013, o. S.).

\subsubsection{Verlernen pädagogisch verhindern wollen}

Verlernen $\mathrm{zu}$ verhindern ist Aufgabe der Gesellschaft, wenn es um die kollektive Geschichte geht: „Das nationale und kollektive Gedächtnis ist fixiert auf etwas, was sich nicht vergessen lässt; es ist fixiert auf nationale Katastrophen und kollektive Kränkungen, zuletzt und unvergesslich auf das Trauma der Hitlerherrschaft" (Prange 2006, S. 233). Die Verhinderung eines Vergessens - hier zeigt sich ein weiteres Mal die Notwendigkeit einer komparativen Analyse der Begriffe „Verlernen“ und „Vergessen“ wird in Anbetracht der historischen Ereignisse zur nationalen Aufgabe. Aber auch auf der Mesoebene, z. B. der eines Klassenverbands, wird Verlernen vor dem Hintergrund didaktischer Überlegungen zu verhindern versucht, beispielsweise durch den Einsatz von Methoden, die ein Festigen und Sichern von Wissen und Fertigkeiten bewirken.

\section{Fazit}

Alltagsweltlich haben wir alle eine Vorstellung davon, was unter den Begriffen ,Vergessen“ und „Verlernen“ zu verstehen ist. Ein erziehungswissenschaftlich fundierter Begriff des Verlernens liegt jedoch bislang nicht vor. Die Re-Analyse unter der Fragestellung, inwieweit ein alltagsweltliches Begriffsverständnis in die Analyse empirischen Materials eingebracht werden kann, offenbarte neben methodischen Schwierigkeiten zugleich ein ganzes Arbeitsprogramm für eine grundlagentheoretische und empirisch fundierte Auseinandersetzung mit Phänomenen des Verlernens. Bereits der Vergleich einiger weniger Arbeiten, die die Begriffe „Verlernen“ oder „Vergessen“" nutzen, zeigt die Vielgestaltigkeit des Verlernens auf: bewusst oder unbewusst; pädagogisch intendiert oder nicht pädagogisch intendiert. Die ausgewählten Beispiele zeigen zudem, dass sich ein Verlernen/Vergessen auf Wissen, Fertigkeiten oder Identität beziehen kann und dass Prozesse des Verlernens/Vergessens auf der Mikroebene (Individuum), der Mesoebene (Organisation) oder der Makroebene (Gesellschaft) ablaufen können.

Um mögliche weitere Verständnisdimensionen des Begriffs „Verlernen“ in den Blick nehmen zu können, ist es notwendig, Thematisierungen des Verlernens auch 
über die eigenen fachdisziplinären Grenzen hinaus (z. B. in Psychologie, Soziologie, Medizin) zu untersuchen. Auch das Verhältnis von Verlernen und Vergessen muss explizit erörtert werden. Nur so kann anschließend eine äußere Abgrenzung zu anderen Lernmodi und eine innere Differenzierung des Verlernens vorgenommen werden. Eine hierfür vorzunehmende empirische Untersuchung kann grundlagentheoretisch erforschen, was Verlernen - ebenso wie Umlernen und Nichtlernen - im Kern ausmacht. In Fortsetzung der begonnenen Arbeiten ist es dann unumgänglich, empirisch kleinschrittig zunächst Phänomene zu betrachten, die Ausdruck eines - wie auch immer gearteten - Verlernens sind. Wird hierfür der Grounded Theory nicht nur methodologisch, sondern auch methodisch gefolgt, müssen mithilfe des offenen Kodierens Kodes generiert werden, die anschließend zu (Kern-)Kategorien zusammengefasst und durch axiales Kodieren, d. h. durch Anlegen des Kodierparadigmas, näher bestimmt werden. Die sich dann abzeichnende Schlüsselkategorie wird durch selektives Kodieren unter Verwendung der Bedingungsmatrix definiert. Dieser Kodierprozess wird letztlich dazu führen, dass die Bezeichnung der Schlüsselkategorie kaum mehr mit dem alltagsweltlichen Begriff des Verlernens zusammenfällt, d. h. die Einführung eines wissenschaftlichen Begriffs möglich wird.

Open Access Dieser Artikel unterliegt den Bedingungen der Creative Commons Attribution License. Dadurch sind die Nutzung, Verteilung, und Reproduktion erlaubt, sofern der/die Originalautor/en und die Quelle angegeben sind.

\section{Literatur}

Baecker, D. (2003). Organisation und Management. Frankfurt a. M.: Suhrkamp Verlag.

Bateson, G. (1985). Ökologie des Geistes. Frankfurt a. M.: Suhrkamp Verlag.

Benjamin, W. (1980). Berliner Kindheit um Neunzehnhundert. In W. Benjamin (Hrsg.), Gesammelte Schriften. Band IV 1: Kleine Prosa, Baudelaire-Übertragungen. Werkausgabe Bd. 10 (S. 235-304). Frankfurt a. M.: Suhrkamp Verlag.

Buck, G. (1989). Lernen und Erfahrung - Epagogik. Zum Begriff der didaktischen Induktion. Darmstadt: Wissenschaftliche Buchgesellschaft.

Dewey, J. (1994). Erziehung durch und für Erfahrung. Stuttgart: Klett-Cotta/J. G. Cotta'sche Buchhandlung Nachfolger.

Dewey, J. (1995). Erfahrung und Natur. Frankfurt a. M.: Suhrkamp Verlag.

Dinkelaker, J. (2011). Lernen. In J. Kade, W. Helsper, C. Lüders, B. Egloff, F.-O. Radtke, \& W. Thole (Hrsg.), Pädagogisches Wissen. Erziehungswissenschaft in Grundbegriffen (S. 133-139). Stuttgart: Kohlhammer Verlag.

Duden. www.duden.de/rechtschreibung/verlernen. Zugegriffen: 05. Dez. 2014.

Giese, C. (1998). Lernen als Erinnern? Strukturelle Überlegungen zum Verhältnis von Lernen und Gedächtnis. Unveröffentlichte Hausarbeit zur Erlangung des Grades einer Magistra Artium. Fakultät für Philosophie, Pädagogik und Publizistik der Ruhr-Universität Bochum.

Glaser, B. G., \& Strauss, A. L. (2008). Grounded Theory. Strategien qualitativer Forschung. Bern: Hans Huber Verlag.

Göhlich, M., \& Zirfas, J. (2007). Lernen. Ein pädagogischer Grundbegriff. Stuttgart: Kohlhammer Verlag.

Göhlich, M., Wulf, C., \& Zirfas, J. (2007). Pädagogische Theorien des Lernens. Weinheim: Beltz Juventa Verlag.

Kade J., \& Seitter, W. (Hrsg.). (2007). Umgang mit Wissen. Recherchen zur Empirie des Pädagogischen. Opladen: Verlag Barbara Budrich.

Knott, M. L. (2011). Verlernen. Denkwege bei Hannah Arendt. Berlin: Verlag Matthes \& Seitz.

Koch, L. (1988). Überlegungen zum Beruf und zur Logik des Lernens. Zeitschrift für Pädagogik, 3, 315-330. 
Koch, L. (1991). Logik des Lernens. Weinheim: Deutscher Studienverlag.

Laertius, D. (1990). Leben und Meinungen berühmter Philosophen. Hamburg: Verlag F. Meiner.

Maier-Gutheil, C. (2009). Zwischen Beratung und Begutachtung: Pädagogische Professionalität in der Existenzgründungsberatung. Wiesbaden: VS Verlag für Sozialwissenschaften.

Meyer-Drawe, K. (2008). Diskurse des Lernens. München: Wilhelm Fink Verlag.

Nittel, D. (2010). Lernphänomene im Kontext lebensbedrohlicher Erkrankungen im Alter. Mechanismen der Erzeugung von biographischer Irrelevanz bei Brustkrebspatienten. In C. Hof, J. Ludwig, \& B. Schäffer (Hrsg.), Erwachsenenbildung im demographischen und sozialen Wandel (S. 94-104). Baltmannsweiler: Schneider Verlag.

Nittel, D. (2011). Die Aneignung von Krankheit: Bearbeitung lebensgeschichtlicher Krisen im Modus des Lernens. Der pädagogische Blick. Zeitschrift für Wissenschaft und Praxis in pädagogischen Berufen, $2,80-90$.

Nittel, D. (2013). Prozessuale Lerndimensionen: Ein biographieanalytisches Instrument zur Beobachtung von Bildungsprozessen bei Menschen mit lebensbedrohlichen Erkrankungen und zur Begründung pädagogischer Interventionen. In H. Herzberg \& A. Seltrecht (Hrsg.), Der soziale Körper. Interdisziplinäre Zugänge zur Leiblichkeit (S. 107-153). Opladen: Verlag Barbara Budrich.

Nittel, D., \& Seltrecht, A. (2013). Krankheit: Lernen im Ausnahmezustand? Brustkrebs und Herzinfarkt aus interdisziplinärer Perspektive. Mit Online-Material für Fachleute. Berlin: Springer Verlag.

Petzelt, A. (1961). Über das Lernen. In A. Petzelt, W. Fischer, \& M. Heitger (Hrsg.), Einführung in die pädagogische Fragestellung. Aufsätze zur Theorie der Bildung. Teil 1 (S. 73-92). Freiburg: Lambertus Verlag.

Platon. (1991). Der Staat. (Politeia). Stuttgart: Reclam Verlag.

Prange, K. (2006). Vergessen - verschweigen - verarbeiten. Zur pädagogischen Reaktion auf die Erfahrung der Hitlerzeit bei Fritz Blättner, Otto Friedrich Bollnow und Theodor Wilhelm. In K.-P. Horn, M. Ogasawara, M. Sakakoshi, H.-E. Tenorth, J. Yamana, \& H. Zimmer (Hrsg.), Pädagogik im Militarismus und Nationalsozialismus. Japan und Deutschland im Vergleich (S. 233-241). Bad Heilbrunn: Klinkhardt Verlag.

Richter, S., \& Friebertshäuser, B. (2013). Brustkrebs als Statuspassage: Leben und Sterben als Krise und Lösung. In D. Nittel \& A. Seltrecht (Hrsg.), Krankheit: Lernen im Ausnahmezustand? Brustkrebs und Herzinfarkt aus interdisziplinärer Perspektive. Mit Online-Material für Fachleute (S. 315-326). Berlin: Springer Verlag.

Ritter, H. (2013). Über das Verlernen. www.faz-net.de. Zugegriffen: 08. Dez. 2014.

Schütze, F. (1981). Prozessstrukturen des Lebensablaufs. In J. Matthes, A. Pfeifenberg, \& A. Stosberg (Hrsg.), Biographie in handlungswissenschaftlicher Perspektive (S. 67-156). Nürnberg: Eigenverlag.

Seltrecht, A. (2013). Eine Familie - eine Geschichte? Argumentationsanalyse familial (un-)sichtbarer Leidens- und Lernprozesse. In H. Herzberg \& A. Seltrecht (Hrsg.), Der soziale Körper. Interdisziplinäre Zugänge zur Leiblichkeit (S. 155-186). Opladen: Verlag Barbara Budrich.

Siewert, A. (2014). ,Unternehmertum “ als lebensgeschichtlich situierter Lernprozess. Eine qualitativrekonstruktive Untersuchung. Unveröffentlichte Dissertation am Fachbereich Erziehungswissenschaften der Goethe-Universität Frankfurt a. M.

Strauss, A. L. (1998). Grundlagen qualitativer Sozialforschung. München: Wilhelm Fink Verlag.

Strauss, A. L., \& Corbin, J. (1996). Grounded Theory: Grundlagen Qualitativer Sozialforschung. Weinheim: Beltz Verlag. 